\title{
Editorial
}

\section{How Good Is the Tuberculin Skin Test?}

\author{
John B. Bass, Jr., MD
}

There may be nostalgia for the days when all tuberculin reactions meant tuberculous infection, and no nonsense.-Edwards and Edwards ${ }^{1}$

Purified protein derivative is a relatively crude material containing several different antigens. The results of a skin test with purified protein derivative are dependent on the immunologic reactivity of the recipient of the test, and the final interpretation is dependent on the individual interpreting the test. Despite these limitations, the tuberculin skin test (TST) is one of the most accurate medical tests available. Using conservative assumptions, I have estimated the sensitivity of the TST at approximately $95 \%$ and the specificity at approximately $99 \%$ to $99.5 \%{ }^{2}$ If these assumptions are correct, the TST is more accurate than most tests commonly used in clinical medicine.

Despite this high degree of accuracy, the TST is not good enough to give valid results in serial skin testing programs in most hospitals and other medical facilities where the prevalence of new infection is low (including facilities ordinarily considered to be at "moderately high risk"). The table translates the conversion rate in a serial skin testing program to the actual transmission rate of tuberculosis infection assuming a sensitivity of 0.95 and a specificity of 0.99 to 0.995 . If the annual conversion rate in a skin testing program is less than $1 \%$, most conversions will be false positives and the predictive value of an individual conversion will be poor. Conversion rates above $2 \%$ increase the predictive value of the test to greater than $50 \%$, and results in skin testing programs with annual conversions of greater that $2 \%$ probably represent mostly true transmission of tuberculous infection. Conversion rates between $1 \%$ and $2 \%$ probably represent equal numbers of false-positive and true-positive tuberculin reactions.

This issue of Infection Control and Hospital
TABLE

Range of ACtual Transmission Rates for Observed Tuberculin Skin Test Conversion Rate*

\begin{tabular}{lc}
\hline Conversion Rate & Actual Transmission Rate (\%) \\
\hline 0.5 & $\sim 0$ \\
1 & $0-0.5$ \\
2 & $0.5-1$ \\
5 & $4-4.5$ \\
\hline *Adapted with permission from Tuberculosis in the Workplace (2001) by the National Academy \\
of Sciences, courtesy of the National Academies Press, Washington, DC. \\
\hline
\end{tabular}

Epidemiology presents four reports concerning the TST. ${ }^{3-6}$ These reports are welcome, as the results of most serial skin testing programs in medical environments go unreported. Cook et al. ${ }^{4}$ report TST results among healthcare workers in New York City. The conversion rate in their study was approximately $1.3 \%$ annually-a rate that suggests equal numbers of true-positive and false-positive conversions. The presence of some true-positive conversions is supported by the increased conversion rate in high-risk settings and among foreign-born individuals, who would be expected to have a higher than average risk in their community away from the workplace. Some of the increased conversion rate among high-risk workers was probably the result of semiannual testing in this group compared with annual testing for low-risk workers, as more frequent testing will increase the cumulative false-positive rate if such a rate is constant for each test. ${ }^{2}$

Garber et al. ${ }^{3}$ report results of skin testing programs in 19 microbiology laboratories in New York City. The incidence of TST conversion in their study of $1 \%$ probably slightly favors false conversions over true conversions, 
although the presence of some true conversions is again strengthened by the increased conversion rate among foreign-born individuals and those who had received bacille Calmette-Guérin vaccination.

Both of these studies included TST reactions of 10 to $14 \mathrm{~mm}$ as a positive result. We have previously reported ${ }^{7}$ that only 3 (17\%) of 18 employees at our institution with TST conversions of 10 to $14 \mathrm{~mm}$ had positive reactions on retesting 1 to 3 years later, whereas $6(67 \%)$ of 9 individuals with conversion reactions greater than $15 \mathrm{~mm}$ had positive results on repeat skin testing. None of our employees at that time had been born in a foreign country or had received bacille Calmette-Guérin vaccination. We postulated that these results were likely due to cross-reactivity to non-tuberculous mycobacteria. In this issue of Infection Control and Hospital Epidemiology, Marsh et al. ${ }^{6}$ confirm our finding that a minority of converted reactions in the range of 10 to $14 \mathrm{~mm}$ will remain positive on retest. In addition, they show that many of these appear to be cross-reactions to non-tuberculous mycobacteria as judged by dominant TST reactions to antigens from Mycobacterium avium.

Lambert et al. ${ }^{5}$ report results and analyze costs of skin testing programs in four hospitals and two health departments. The conversions in hospitals 1 and 3 and health department 1 were probably largely, if not entirely, false-positive conversions. Health department 2 probably had a predominance of false conversions, but 0 to 4 true conversions could be expected. Hospital 4 probably had a slight predominance of true conversions and the range of expected true conversions would be 10 to 19 . Hospital 2 is the only program that probably had a significant predominance of true conversion reactions (positive predictive value, $60 \%$ to $80 \%$ ).

If the results from the four hospital programs and two health department programs are combined, the range of overall true conversions is approximately 17 to 33 , with an overall cost of $\$ 2,119,037$. Thus, the cost of detecting a single true conversion ranges from $\$ 64,000$ to $\$ 125,000$. If one-third of the converters complete preventive therapy, the cost per individual completing therapy ranges from $\$ 205,000$ to $\$ 375,000$. If the lifelong efficacy of a completed course of preventive therapy is $70 \%$, the cost of preventing a case of tuberculosis ranges from $\$ 293,000$ to $\$ 535,000$. In addition, there would be 2 to 3 false-positive conversions for every true conversion, resulting in unnecessary therapy with no potential benefit. Also, none of the TST conversions in these programs could be interpreted accurately in real time-only after all the data were collected and assessed at the end of the year could the predictive value of a positive TST be estimated. The TST, despite its outstanding operating characteristics, is not good enough to be used for routine surveillance in most medical environments.

\section{REFERENCES}

1. Edwards PQ, Edwards LB. Story of the tuberculin test. American Review of Respiratory Diseases 1960;81:1-49.

2. Bass JB. The tuberculin skin test. In: Field MJ, ed. Tuberculosis in the Workplace. Washington, DC: National Academies Press; 2001:179-188.

3. Garber E, San Gabriel P, Lambert L, Saiman L. A survey of latent tuberculosis infection among laboratory healthcare workers in New York City. Infect Control Hosp Epidemiol 2003;24:801-806.

4. Cook S, Maw KL, Munsiff SS, Fujiwara PI, Frieden TR. Prevalence of tuberculin skin test positivity and conversions among healthcare workers in New York City during 1994 to 2001. Infect Control Hosp Epidemiol 2003;24:807-813.

5. Lambert L, Rajbhandary S, Qualls N, et al. Cost of implementing and maintaining a tuberculin skin test program in hospitals and health departments. Infect Control Hosp Epidemiol 2003;24:814-820.

6. Marsh BJ, San Vicente J, von Reyn CF. Utility of dual skin tests to evaluate tuberculin skin test reactions of 10 to $14 \mathrm{~mm}$ in healthcare workers. Infect Control Hosp Epidemiol 2003;24:821-824.

7. Bass JB, Sanders RV, Kirkpatrick MB. Choosing an appropriate cutting point for conversion in annual tuberculin skin testing. American Review of Respiratory Diseases 1985;132:379-381. 